EESTI NSV TEADUSTE AKADEEMIA TOIMETISED 1953. II k., nr. 3 ИЗВЕСТИЯ АКАДЕМИИ НАУК ЭСТОНСКОИ ССР 1953. Том II, 쇼 3

\title{
О ГРАВИТАЦИОННОМ ПОТЕНЦИАЛЕ ГАЛАКТИКИ И ТРЕТЬЕМ ИНТЕГРАЛЕ ДВИЖЕНИЯ ЗВЕЗД *
}

\author{
Г. Г. кузмин, \\ кандидат физико-математических наук
}

Одной из основных задач практической звездной динамнки является определение гравитапионного потенциала Галактики и распределения масс в ней. Выдающнеся успехи советских астрономов Б. В. Кукаркина, П. П. Паренаго и др.(1) в выявлении подсистем Галактики и в изучении их пространственно-кинематической структуры позволили сделать первые серьезные шаги в направлении решения упомянутой задачи. Мы имеем в виду прежде всего последние работы П. П. Паренаго по практической звездной динамике, поставившие эту задачу и решившие ее в первом приближении $(5,6)$. Работы П. П. Паренаго вызвали большой ннтерес к вопросам практической звездной динамики. Почти одновременно с последними работами Паренаго в этой области автором настоящей статьи были опубликованы работы, также касающиеся вопроса распределения масс в Галактике и ее гравитационного потенциала $(1,2)$. Продолжая работу в этом направлении, автору удалось получить еще некоторые новые результаты, в частности по вопросу о третьем интеграле движения звезд и связанным с ним вопросам строения Галактики. Цель настоящей статьи - дать краткий критический обзор по данной проблеме, с приведением ряда новых результатов.

При определении гравитационного потенциала Галактики исходными наблюдательными данными являются, во-первых, данные о вращении подсистем Галактики и, во-вторых, данные о двнжении звезд перпендикулярно галактической плоскости и о распределении их пространственной плотности в этом направлении.

Данные о вращении подсистем Галактики служат для определения зависимости потенциала от расстояния до-оси Галактики $R$. В случае плоских подсистем скорость вращения практически равна круговой скорости, по которой получаем радиальное ускорение и, следовательно, радиальный градиент потенциала. Однако для определения радиального ускорения желательно, а иногда и неизбежно, привлечение данных о вращении промежуточных и даже сферических подсистем. Для таких подсистем необходимо применение статистических уравнений движения. Здесь можно воспользоваться соответствующим уравнением Джинса $\left({ }^{7}, 16\right)$, исправив это уравнение за эффект наклонности эллипсонда скоростей $\mathrm{k}$

* Доклад на сессии Аетрономического Совета АН СССР и Ннститута физнки и астрономии АН ЭССР, $27-29$ мая 1953 г. в г. Тарту. 
галактической плоскости. Как мы увидим ниже, существование такой наклонности вне галактической плоскости весьма вероятно. Кроме того, уравнение Джинса можно видоизменить, учитывая соотношение Линдблада-Оорта $(7,18,21)$, связывающее отношение осей эллипсоида скоростей с радиальным градиентом скорости вращения. (Это соотношение можно вывести из таких же довольно общих соображений, как и уравнение Джинса.) Мы получим тогда для галактической плоскости

$$
-R \frac{\partial \Phi}{\partial R}=V^{2}+q \sigma_{R}^{2},
$$

где $\Phi$ - потенциал, $V$ - скорость вращения, $\sigma_{R}-$ дисперсия $R$-компонентов скоростей и

$$
q=-\frac{\partial \ln \left(D \sigma_{R}^{2} \omega^{-1 / 2}\right)}{\partial \ln R}+q_{1} .
$$

В последней формуле $D$ - пространственная звездная плотность, $\omega-$ угловая скорость вращения и $q_{1}-$ член, учитывающий наклонность эллипсоида скоростей. Этот член имеет вйд:

$$
q_{1}=-\left(1-\frac{\sigma_{z}^{2}}{\sigma_{R}^{2}}\right) \frac{R}{R_{0}},
$$

где $\sigma_{z}^{2}-$ дисперсия $z$-компонентов скоростей, $R_{0}-$ расстояние от точки конвергенцин направлений больших полуосей эллипсоида скоростей вблизи галактической плоскости.

Уравнение (1), учитывающее наклонность эллипсоида скоростей к галактической плоскости и радиальный градиент дисперсии $\sigma_{R}$, является более общим, чем обычно применяемое оортовское уравнение асимметрии звездных движений $(7,21)$. Член $q \sigma_{R}^{2}$, определяющий асимметрическое смещенне центронда, пренебрежимо мал для плоских подсистем. Для промежуточных подсистем он носит характер малой поправки, а для сферических подсистем делается преобладающим. Коэффициент $q$ определяется в основном радиальным градиентом пространственной звездной плотности, поскольку градиенты $\sigma_{R}^{2}$ и $\omega^{-1 / 2}$ и член $q_{1}$, вероятно, сравнительно невелики. Член $q_{1}$ очень трудно определить из наблюдательных данных; приходится ограничиваться его приближенной оценкой, нсходя из теоретических соображений.

Данные о вращении подсистемы долгопериодических цефеид, исследованном П. П. Паренаго $\left({ }^{8}\right)$, позволяют довольно уверенно установить ход радиального ускорения от $R=5$ кпс до $R=10$ кпс. Для $R>10$ кпс данные для определения радиального ускорения практически отсутствуют. Для $R<5$ кпс оно определяется лишь приближенно из данных о вращении подсистемы планетарных туманностей и промежуточной подсистемы долгопериодических цефеид * и данных о пространственно-кинематическом строении сфернческих подсистем. В случае последних радиальное ускорение определяет в основном второй член в формуле (1), который можно приближенно оценить. Следует заметить, что этот член довольно уверенно определяется для окрестностей Солнца, что позволяет определить круговую скорость в окрестностях Солнца независимо от оортовских постоянных и расстояния Солнца от центра Галактики. Такое определе-

* Как известно, часть долгоперноднческнх цефенд имеет большую дисперсию скоростей. Судя по последней и по скорости центронда, эти цефенды принадлежат не к сферической подсистеме, а к промежуточной, как и планетарные туманности. 
ние круговой скорости было сделано П. П. Паренаго $\left({ }^{9}\right)$ при помощи оортовской формулы асимметрии. Результат подтверждает оценку, получаемую по оортовским постоянным, и устраняет некоторую неуверенность, возникшую в связи с неуверенностью постоянной $B$.

Кривая круговой скорости вращения Галактики представлена на pнс. 1. Она взята из нашей работы $\left({ }^{2}\right)$. Сплошная часть кривой соответствует несколько сглаженным данным о вращении подсистемы долгопериодических цефеид в промежутке от $R=5$ кпс до $R=10$ кпс. Экстраполированная и интерполированная части кривой изображены прерывисто. Три точки соответствуют грубым оценкам круговой скорости для малых $R$ (точки с обозначением величнны средней ошибки получены по промежуточным подсистемам, точка в скобках - по сферическим).

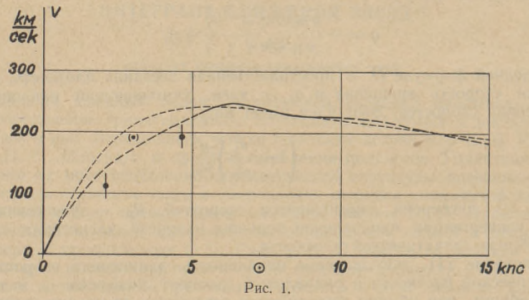

Кривая круговой скорости в хорошо исследованном интервале $R$ имеет заметное искривление и точку перегиба. Ход скорости вращения здесь заметно уклоняется от плавного хода согласно обычно принимаемому линдбладовскому закону вращения $(7,20)$ :

$$
V=\frac{c_{3} R}{c_{1}+c_{2} R^{2}}
$$

где $c_{1}, c_{2}, c_{3}$ - постоянные. Правда, кривая вращения на рис. 1 может быть несколько искажена ошибками в определении расстояний долгопериодических цефеид, возникающими вследствие несовершенного учета поглощения. Однако все же приходится, повидимому, считаться с возможностью реальных уклонений от закона (4) в изученном интервале $R$. Вероятно, этот закон применим лишь как приближенная интерполяционная формула, особенно в случае плоских подсистем. В качестве закона круговой скорости он явно не годится для больших $R$, давая слишком быстрое падение радиального ускорения с увеличением $R$.

Для плоских подсистем упомянутый закон плохо обоснован также теоретически. Он выводится при предположении, что распределение скоростей совершенно точно шварщшильдово или вообще эллипсоидально. Такое допущение можно сделать в математической задаче, но в случае реальной Галактики мы его делать не вправе. Если же от него отказаться, то ничего определенного о законе вращения плоских подсистем вывести нельзя кроме того, что скорость вращения должна практически совпадать с круговой скоростью. Дисперсия скоростей мала и распределение их практически шварцшильдово, если фазовая плотность пропорциональна 
$e^{k l}$, где $k$ - достаточно большая положительная постоянная и $f-$ плавно нзменяющаяся функция интегралов движения, имеющая в пространстве скоростей только один максимум. Что распределенне скоростей получается практическн шварцшильдовым, нетрудно убедиться, разложив функцню $f$ в ряд Тэйлора у максимума фазовой плотности в пространстве скоростей. Так как $k$ велико, то важны лишь первые члены разложения, квадратичные относительно компонентов скоростей. Но в то время, как распределение скоростей получается шваршшильдовым независимо от внда функции $f$, закон вращения оказывается зависимым от этой функции и, не зная ее, нельзя сказать ничего определенного о законе вращения. Правда, на функцню $f$ нужно наложить известные ограничения для того, чтобы радиальный граднент пространственной звездной плотности не был очень большим. Тогда получается, что скорость вращения практически совпадает с круговой скоростью. Однако ограничения, накладываемые на $f$, зависят от закона круговой скорости, а не наоборот, и ничего определенного о законе вращения опять-таки сказать нельзя.

Таким образом, рассматрнваемый закон вращения, с одной стороны, не нмеет достаточных подтверждений наблюдательными данными, а с другой стороны, плохо обоснован теоретнчески. Ввнду этого исследование вращения Галактики, особенно ее плоских подсистем, должно идти по возможности не по линии определения параметров этого закона, а по линии детального изучения зависнмости скорости вращения от $R$ и вывода по ней радиального ускорения и потенщиала.

Нельзя согласиться с П. П. Паренаго, принимающего в свонх работах о потенциале Галактики $(5,6)$ закон (4) для круговой скорости и выводящего отсюда выражение для потенциала в галактической плоскости. Если при малых $R$ этот закон может быть прнменен как приближенная интерполяцнонная формула, то для больших $R$, как уже указывалось, он совершенно негоден. Он дает массу Галактики, равную нулю, поскольку соответствующий ему потенциал стремится к нулю с увеличением $R$ быстрее, чем $R^{-1}$. Из нулевой массы следует, что для некоторых областей пространства плотность материи должна получиться отрицательной. Так оно и оказалось. Для больших расстояний от галактической плоскости Паренаго получил отрицательные плотности.

Слишком быстрое убывание потенциала для болыших $R$, приводящее к нулевой массе Галактики, получается оттого, что радиальное ускорение согласно закону (4) также слишком быстро убывает с увеличением $R$. Но это снижает значения потенциала не только для больших $R$, но и для всех $R$, поскольку потенцнал получается интегрированием радиального ускорения от заданного $R$ до $\infty$. Поэтому результаты, полученные П. П. Паренаго для скорости освобождения (в частности, $300 \mathrm{~km} / \mathrm{cek}$ в окрестностях Солнца), сильно занижены. Это, однако, не значит, что его формула для потенциала Галактики непригодна. Она применима как более или менее приближенная интерполяционная формула для не очень больших расстояний от центра Галактики. Но при этом остается неопределенной некоторая аддитивная постоянная.

Зная раднальное ускорение вблизи галактической плоскости, можно получить уже некоторые данные о распределенни масс в Галактике. Имеется возможность вычислить поверхностную плотность Галактики, т. е. плотность, спроицированную на галактическую плоскость. Аналогичная задача для туманностей Андромеды и Треугольника решалась уже Уайзом и Мэйоллом $\left({ }^{26}\right)$, причем они пользовались моделью плоского диска. Попытка решить задачу для Галактики сделана в нашей вышеупомянутой работе $\left({ }^{2}\right)$, где проблема рассматрнвается в более общем виде, причем учитывается, что Галактика имеет некоторую жтолщину». Учет «толщины» 
Галактики, однако, мало меняет результаты. Еще менее они должны зависеть от того, какой закон будет принят для убывания плотности с удалением от галактической плоскости. Если предположить поверхности равной плотности сферондальными, то поверхностная плотность $\delta$ вычислится по формуле

$$
\delta=\frac{1}{2 \pi} \int_{R}^{\infty} \frac{\mu(a)}{\sqrt{a^{2}-R^{2}}} \frac{d a}{a},
$$

где $\mu(a) d a$ - масса между сфероидами равной плотности с большими полуосями $a$ и $a+d a$. Функция $\mu(a)$, как показано в работе, связана с радиальным ускорением в галактической плоскости уравнением

$$
-\frac{\partial \Phi}{\partial R} R=G \int_{0}^{R} \frac{\mu(a) d a}{\sqrt{R^{2}-a^{2} e^{2}}},
$$

где $G$ - гравитационная постоянная и $e-$ эксцентриситет меридианных сечений сфероидов равной плотности. Если $e=1$, то мы имеем плоскую модель Галактики. В этом случае интегральное уравнение (6) сводится к уравнению типа Абеля, и $\mu(a)$ выражается в квадратурах. Чтобы учесть фтолщину» Галактики, нужно в полученный таким образом результат внести некоторую поправку, выражение для которой дано в той же работе.

Несмотря на то, что данные о радиальном ускорении вблизи галактической плоскости еще далеко не полны, все же можно получить довольно уверенные результаты для поверхностной плотности, особенно в окрестностях Солнца $\left({ }^{2}\right)$. На рис. 2 представлена кривая поверхностной плотности, как она получилась на основании кривой круговой скорости на рис. 1. Вблизи Солнца поверхностная плотность оказывается равной 140 солнечным массам на кв. парсек. Это примерно вдвое превышает результат, полученный в свое время Оортом ${ }^{22}$ ) по звездностатистическим данным с учетом межзвездной матерни. Расхождение, по крайней мере отчасти, объясняется тем, что Оортом не были учтены сферические подсистемы Галактики. Қривая поверхностной плотности на рис. 2 довольно неплохо апроксимируется экспоненциальным законом, причем для радиального градиента логарифма плотности получается $-0,15$ на килопарсек. Результат этот представляется также довольно приемлемым.

Интересно отметить, что детали наиболее достоверной части кривой поверхностной плотности (сплошная часть кривой) воспроизводят детали распределения плотности сферических подсистем $\left({ }^{3}\right)$. Это может служить аргументом в пользу того, что сферические подсистемы играют заметную роль в распределении масс Галактнки.

Кроме поверхностной плотности, данные о радиальном ускорении позволяют оценить полную массу'Галактики и вычислить скорость освобождения для различных $R$ в галактической плоскости. На основании того, что говорнлось выше, ясно, что результаты, полученные для массы и скорости освобождения, существенным образом зависят от того, как экстраполировать круговую скорость на $R>10$ кпс. Во избежание отрицательных плотностей целесообразно провести. это косвенно, экстраполируя функцию $\mu(a)$, которая должна быть положительной и сходить на нет при приближении $a$ к некоторому значению, принимаемому за эффективный радиус Галактики. Результаты, полученные в рассматриваемой работе $\left({ }^{2}\right)$ для массы и скорости освобождения, следующие: масса Галактики - 100 миллиардов солнечных масс, скорость освобождения в окре- 
стностях Солнца - 365 км/сек. Эти результаты хорошо согласуются с результатами В. С. Сафронова $\left({ }^{12}\right)$, получившего их несколько иным путем. Қак и следовало ожидать, скорость освобождения получается значительно большей, чем у Паренаго. Она превышает круговую скорость на $125 \mathrm{kм} /$ сек или $52 \%$.

Можно, пожалуй, возразить, что большая скорость освобождения протнворечит данным об асимметрии звездных движений, на основании которой Оорт $\left({ }^{21}\right)$ заключил, что скорость освобождения превышает кру-

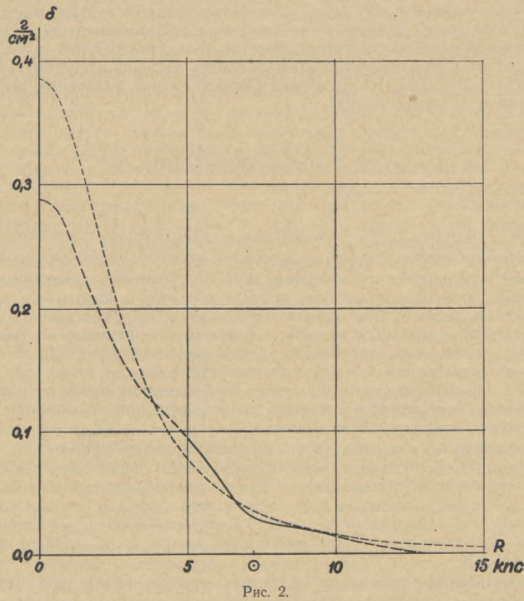

говую скорость всего лишь примерно на 65 км/сек. Однако, как отметил уже Боттлингер $\left({ }^{13}\right)$, почти полное отсутствие звезд со скоростями, превышающими круговую более, чем на 65 км/сек, указывает на ограниченность размеров Галактики, а не на величину скорости освобождения. Поэтому никак нельзя согласиться с авторами, принимающими за разность скорости освобождения и круговой скорости упомянутое значение. Что оно слишком мало, видно уже из того, что Перек $\left({ }^{23}\right)$, приняв его в своих вычислениях, получил совершенно неприемлемые результаты о местоположении Солнца в Галактике. Солнце оказалось у него находящимся на самой периферии системы.

До сих пор мы рассматривали вопрос о зависимости гравитационного потенциала Галактики от расстояния до ее оси и о данных, которые 
можно получнть о Галактике, зная эту зависимость. Обратимся теперь к вопросу о зависимости потенциала от расстояния до галактической плоскости. Для изучения этой завнсимости нужно применить данные о движении и пространственном распределении звезд в направлении, перпендикулярном галактической плоскости. При обработке этих данных можно было бы опять воспользоваться соответствующим статистическим уравнением Джинса $(7,16)$. Однако применение уравнения Джинса затруднительно, поскольку для этого требуются данные о дисперсии z-компонентов скоростей на различных расстояниях от галактической плоскости, а такие данные пока отсутствуют. Но упомянутое затруднение можно обойти, применив данные о распределении $z$-компонентов скоростей вблизи галактической плоскости. Это было сделано Оортом в его известной работе о притяжении в направлении, перпендикулярном галактической плоскости $\left({ }^{22}\right)$. По сути дела Оорт применил уравнение, выведенное нами в следующем виде (1):

$$
D=\int_{-\infty}^{\infty} F\left[v_{z}^{2}-2\left(\Phi-\Phi_{0}\right)\right] d v_{z}
$$

Здесь $\Phi$ - потенциал, $\Phi_{0}$ - потенциал в галактической плоскости, $v_{z}-$ $z$-компонент скорости, $F\left(v_{z}^{2}\right)$ - функция распределения $z$-компонентов скорости в галактической плоскости и $D$ - пространственная плотность звезд. При этом предполагается, что $D, F, \Phi$ и $\Phi_{0}$ относятся к одному и тому же значению $R$. Если функция $F$ известна, то уравнение $(7)$ позволяет вычислить пространственную плотность как функцию от разности $\Phi-\Phi_{0}$, откуда, зная зависимость пространственной плотности от галактического возвышения $z$, найдем упомянутую разность также как функцию от $z$. При этом, как мы видим, нет необходимости прибегать к последовательным прнближениям, как это делает Оорт, привлекающий в своих вычислениях также уравнение Джинса.

Уравнение (7) выведено в предположении стационарности и осевой симметрии Галактики. Наклонность эллипсонда скоростей к галактической плоскости не учитывалась. Такая наклонность должна вызвать явную зависимость функции $F$ от $z$. Но можно показать, что для плоских подсистем эта зависимость не играет существенной роли. Для промежуточных и сферических подсистем ее роль может быть, однако, весьма значительной.

Для отдельных подсистем Галактики можно считать распределение z-компонентов скоростей приблизительно гауссовым. Тогда уравнение (7) принимает вид:

$$
D=D_{0} e^{\frac{\Phi-\Phi_{0}}{\sigma_{z}^{2}}},
$$

где $D_{0}$ - пространственная плотность звезд в галактической плоскости и $\sigma_{z}$ - дисперсия $z$-компонентов скоростей, которая в этом случае не зависит от $z$. Вблизи галактической плоскости уравнение превращается в следующее:

$$
D=D_{0} e^{-\frac{z^{2}}{2 \zeta^{2}}},
$$


где величина Ђ связана с $\sigma_{z}$ соотношением

$$
\frac{\sigma_{z}}{\zeta}=C, \quad C^{2}=-\left[\frac{\partial^{2} \Phi}{\partial z^{2}}\right]_{z=0}
$$

Уравнения (8) и (9) вытекают также из уравнения Джинса, если принять $\sigma_{z}$ незавнсимым от $z$. Они не учитывают наклонности эллипсонда скоростей к галактической плоскости, что важно иметь в виду, особенно в случае промежуточных и сфернческих подсистем.

Ход потенциала в нанболее широком интервале значений z был исследован П. П. Паренаго $\left({ }^{6}\right)$, применившим уравненне (8) и использовавшим данные Б. В. Кукаркина о пространственных плотностях цефеид и мирид $\left({ }^{3}\right)$. Так как значения, которые были приняты для $\sigma_{z}$, довольно неуверенны, а наклонность эллипсоида скоростей к галактической плоскости н возможный граднент $\sigma_{z}$ по $z$ не учитывалисьь, то полученные результаты нельзя считать пока еще вполне уверенными. Значительно более точные результаты можно в настоящее время получить для потенциала вблизи галактической плоскости, определяя постоянную $C$ в окрестностях Солнца. Здесь могут быть привлечены данные для целого ряда подсистем Галактики. Такая работа была выполнена еще Оортом в его вышеупомянутом исследовании $\left({ }^{22}\right)$. Но использованные им данные наблюдений уже в известной степени устарели, и постоянная $C$ требует переопределения. Шаги в этом направлении были сделаны в нашей уже упоминавшейся работе (1). Обработав данные о собственных движениях А-звезд и К-гигантов вблизи галактического экватора, мы получили $C=$ $=56 \pm 5$ км/сек на килопарсек. Это заметно меньше результата Оорта, согласно которому $C=73 \mathrm{kм} /$ сек на килопарсек. Величина $C$ определена также П. П. Паренаго $\left({ }^{6}\right)$ по цефеидам и миридам, причем он получил для $C$ значение, близкое к оортовскому. Однако его результат представляется нам менее уверенным, чем наш. Для определения $C$ ему пришлось данные Б. В. Кукаркина $\left({ }^{3}\right)$ о плотностях короткопериодических цефеид и мирид экстраполировать на малые $z$, а это можно было сделать лишь очень приближенно. Правда, для малых $z$ имелись данные о пространственных плотностях долгопериодических цефенд, но зато для этих звезд очень неуверенно определяется $\sigma_{z}$. Впрочем и наше значение $C$ не очень уверенно и может быть преуменьшенным вследствие некоторых не учтенных нами систематических ошибок. Тем не менее представляется все же вероятным, что $C$ несколько меньше оортовского значения.

Если известна зависимость потенциала от $R$ и от $z$, то можно вычислить пространственную плотность материи при помощи уравнения Пуассона. Введя оортовские постоянные $A$ и $B$ для движения по круговым орбитам, получим это уравнение для окрестностей галактической плоскости в виде

$$
4 \pi G \varrho=C^{2}-2\left(A^{2}-B^{2}\right),
$$

где $\varrho$ - плотность. Формула эта была выведена Линдбладом $\left({ }^{19}\right)$. Позднее она была независимо от Линдблада выведена П. П. Паренаго $\left({ }^{6}\right)$ и автором $\left({ }^{1}\right)$ и, повидимому, впервые применена на практике. Оорт в своей вышеуказанной работе $\left({ }^{22}\right)$, как известно, также вычислял плотность материн. Но он не воспользовался уравнением Пуассона, а предпочел вычислять плотность при помощи моделей Галактики, состоящих из однородных сферондов. Такой же метод применил впоследствии В. С. Сафронов $\left({ }^{12}\right)$.

В упомянутой выше работе (1) мы получнли при помощи формулы (11) для плотности в окрестностях Солнца $0,05 \pm 0,01$ солнечной массы 
на куб. парсек. Результат этот приблизительно вдвое меныше результата Оорта, что связано с меньшим значением постоянной $C$. Полученная плотность лишь немногим превышает плотность, обусловленную звездами. Но поскольку наше значение $C$ несколько преуменышено, то действительная плотность должна быть больше, хотя она все же, вероятно, не достигает оортовского значения.

Плотность материи в окрестностях Солнца определялась также Паренаго $\left({ }^{6}\right)$, Сафроновым $\left({ }^{12}\right)$, Схилтом $\left({ }^{24}\right)$ и другимн. Схилт получил совершенно абсурдный результат, свидетельствующий о том, что примененный им способ решения задачи непригоден. Результаты же Паренаго и Сафронова близки к оортовскому. Это согласие, однако, еще не подтверждает правильности результата Оорта. Значение $C$, полученное Паренаго, как уже говорилось, очень неуверенно, а Сафронов пользовался оортовским значением $C$, не определяя этой постоянной заново. Правда, Сафронов вычислял плотность не только с оортовским значением $C$, но и независимо от него, по данным о галактическом вращении, получив при этом плотность, опять-таки близкую к оортовской. Но это указывает лишь на то, что принятая Сафроновым сплюснутость Галактики хорошо согласуется с оортовским значением $C$. Сплюснутость же Галактики является величиной, которая сама подлежит определению из динамических соображений.

Для определения сплюснутости Галактики могут быть применены данные о пространственной плотности вблизи галактической плотности и о поверхностной плотности Галактики, позволяющие оценить эквивалентную полутолщину Галактики. Согласно нашим результатам для этих величин, эквивалентная полутолщина Галактики в окрестностях Солнца получается равной 1,4 килопарсека $\left({ }^{2}\right)$. Результат этот, без сомнения, преувеличен, и не только потому, что пространственная плотность получилась у нас слишком малой, но и потому, что наш результат для поверхностной плотности, повидимому, также немного преувеличен. Тем не менее следует считать вполне вероятным, что эквивалентная полутолщина Галактики достигает в окрестностях Солнца по меньшей мере одного килопарсека. Если предположнть Галактику сферондальной, то такая эквивалентная полутолщина соответствует отношению полуосей, равному приблизительно $1 / 5$. Таким образом, сплюснутость Галактики оказывается довольно незначительной. Повидимому, можно считать, что сплюснутость Галактики значительно меньше, чем принималась до сих пор, и что масса сферических подсистем Галактики довольно велика. Такой вывод согласуется с результатом П. П. Паренаго $\left({ }^{10}\right)$ о большой численности субкарликов и С. Б. Пикельнера ('1) о прннадлежности значительной доли межзвездного газа к сферической подсистеме.

Выше мы рассмотрели вопрос об определении потенциала Галактики и распределении масс в ней. Нам остается еще изложить некоторые результаты о возможном строении Галактики в связи с третьим интегралом движения звезд. Как известно, одной из трудностей динамики стационарной Галактики является объяснение трехосности эллипсонда скоростей. Обычно, согласно Джинсу $\left({ }^{17}\right)$, считается, что фазовая плотность звезд является функщией двух интегралов движения - интеграла энергин $I_{1}$ и интеграла импульса вращения вокруг оси Галактики $I_{2}$. Но в таком случае оказывается, что распределение скоростей должно иметь симметрию относительно оси, расположенной в направлении вращения Галактики. В действительности же распределение скоростей трехосное, причем нанбольшая ось эллипсоида скоростей расположена более или менее радиально. Однако эту трудность можно устранить, если принять 
во внимание еще третнй интеграл движения звезд. В качестве такого интеграла Линдблад $\left({ }^{20}\right)$ предложил интеграл энергии по z-координате:

$$
I_{3}=v_{2}^{2}-2\left(\Phi-\Phi_{0}\right) \text {. }
$$

Этот интеграл, строго говоря, является интегралом движения лишь тогда, когда потенциал представляет собой сумму функции от $R$ и функции от $z$, т. е. когда $\partial^{2} \Phi / \partial R \partial z=0$. Для Галактики в целом упомянутое условие, конечно, не выполняется; но если ограничнться окрестностями галактической плоскости и малым ннтервалом значений $R$, то это условие можно считать выполненным с достаточной точностью. Поэтому для звезд, движущихся по орбитам, близким к круговым, $I_{3}$ остается практически постояныым. Отсюда следует, что для звезд плоских подсистем можно счнтать фазовую плотность функцией не только от $I_{1}$ и $I_{2}$, но и от $I_{3}$. Распределение скоростей для плоских подсистем получится тогда трехосным.

Однако распределение скоростей трехосно не только для плоских, но и для промежуточных и сферическнх подсистем. Для последних интеграл Линдблада уже не пригоден и нужно искать третий интеграл в более общем виде. Так как распределение скоростей для отдельных подснстем приблизительно шваршшильдово, то естественно потребовать, чтобы третий интеграл допускал шварцшильдово распределение скоростей. Тогда его следует искать в виде выражения, квадратичного относительно компонентов скоростей. Если предположить третий интеграл независимым от галактоцентрической долготы, то из условия неизменности значения интеграла при движении звезды нетрудно найти $I_{3}$ в следующем виде:

$$
I_{\mathrm{s}}=\left(R v_{z}-z v_{R}\right)^{2}+z^{2} v_{\theta}^{2}+z_{0}^{2}\left(v_{z}^{2}-2 \Phi^{*}\right),
$$

где $v_{R}, v_{z}$ и $v_{\theta}$ соответственно $R$-, $z$ - и $\Theta$-компоненты скорости, $z_{0}-$ постоянная и $\Phi^{*}$ - функция, удовлетворяющая уравнениям

$$
\frac{\partial \Phi^{*}}{\partial R}=\frac{z^{2}}{z_{0}^{2}} \frac{\partial \Phi}{\partial R}-\frac{R z}{z_{0}^{2}} \frac{\partial \Phi}{\partial z}, \quad \frac{\partial \Phi^{*}}{\partial z}=\left(1+\frac{R^{2}}{z_{0}^{2}}\right) \frac{\partial \Phi}{\partial z}-\frac{R z}{z_{0}^{2}} \frac{\partial \Phi}{\partial R} .
$$

Первые два члена полученного интеграла представляют собой квадрат компонента импульса вращения, направленного параллельно галактической плоскости. Последний же член имеет форму интеграла энергии по z-координате, но вместо разности потенциалов здесь фигурирует функция $\phi^{*}$.

Функщия $\Phi^{*}$ должна удовлетворять одновременно обоим уравнениям (14). Поэтому $\partial^{2} \Phi^{*} / \partial R \partial z$ должно получиться из обоих уравнений одинаковым. Отсюда вытекает условие совместимости этих уравнений и, вместе с тем, условие существования интеграла (13); оно имеет вид:

$$
3\left(z \frac{\partial \phi}{\partial R}-R \frac{\partial \Phi}{\partial z}\right)-\left(R^{2}+z_{0}^{2}-z^{2}\right) \frac{\partial^{2} \Phi}{\partial R \partial z}+R z\left(\frac{\partial^{2} \Phi}{\partial R^{2}}-\frac{\partial^{2} \Phi}{\partial z^{2}}\right)=0 .
$$

Это условие совпадает по своей форме с условием, известным из общей динамики $\left({ }^{25}\right)$, как условие существования при плоском движении интеграла, квадратичного относительно компонентов скоростей. Оорт в своей известной работе по динамике Галактики $\left({ }^{21}\right)$ получил также это условие как условие трехосности шварцшильдова распределения скоростей. Он считал условие невыполненным и пришел к заключению, что теория допускает лишь двуосное распределение скоростей. Наблюдаемое трехоеное распределение скоростей он объяснил тем, что движение звезд пронсходит практически так, как если бы $\partial^{2} \Phi / \partial R \partial z=0$. Но это объяснение пригодно, как мы видели, только в случае плоских подсистем. Для объяснения 
же трехосности распределения скоростей промежуточных и сферических подсистем нужно нсходить нз более общего условия (15). Если считать, что оно с достаточной точностью выполняется при движении звезд этих подсистем, то фазовую плотность звезд можно считать функцией от $I_{1}$, $I_{2}$ и $I_{3}$, и распределение скоростей получится трехосным.

Условие (15) является по сравнению с условием $\partial^{2} \Phi / \partial R \partial z=0$ более общим и более легко выполнимым, потому что оно включает произвольную постоянную $z_{0}$, которую можно подобрать в соответствии с характером потенциала в той области пространства, где пронсходит движение звезды. Так как трехосное распределение скоростей наблюдается даже для сферических подсистем, то можно думать, что $z_{0}$ приблизительно постоянно для весьма обширных областей пространства. Большой интерес представляло бы определение величины $z_{0}$ из наблюдательных данных. Для этого можно было бы воспользоваться формулой, следующей нз условия (15), если его применить для окрестностей галактической плоскости и ввести оортовские постоянные $A$ и $B$ и величину $C$. Эта формула имеет вид:

$$
\left(R^{2}+z_{0}^{2}\right) \frac{d C^{2}}{d R}=-4 R\left[C^{2}+B(A-B)\right] .
$$

Определение $z_{0}$ на основании формулы (16) представляет собой, однако, довольно трудно осуществимую задачу, ввиду трудности определения радиального градиента величнны $C$. Ниже $z_{0}$ будет определено другим способом.

В пространстве скоростей интеграл (13) симметричен относительно двух взаимно перпендикулярных плоскостей, пересекающихся по прямой $v_{R}=v_{z}=0$. Так как интеграл $I_{1}$ симметричен относительно этой прямой, а интеграл $I_{2}$ не содержит $v_{R}$ и $v_{z}$, то упомянутые плоскости являются также плоскостями симметрии распределения скоростей. Для угла наклонности этих плоскостей к галактической плоскости имеет место формула

$$
\tan 2 a=\frac{2 R z}{R^{2}+z_{0}^{2}-z^{2}} .
$$

В галактической плоскости или на галактической оси угол $\alpha$ равен нулю или $90^{\circ}$, вообще же он отличается от нуля или $90^{\circ}$. Отсюда следует, что вне галактической плоскости эллипсоид скоростей имеет наклон к последней. Обнаружить этот наклон и другие связанные с ним кинематические эф. фекты весьма трудно при современных наблюдательных возможностях. Теоретически к заключению о наклонности эллипсоида скоростей к галактической плоскости пришел еще Эдлинттон (15) в 1915 г. в своей работе о динамике звездной системы, где он рассматривает возможность трехосного шваршшильдова распределения скоростей в случае стационарности Галактики. Позднее к тому же заключению пришел Чандрасекар (14). Однако анализ работы Чандрасекара показывает, что она содержит ошибки. Методы, применнмые лишь при трактовке движения по орбитам, близким к круговым, используются на больших расстояниях от галактической плоскости, где орбиты резко отличаются от круговых. Делаются заключения об устойчивости кругового движения, не имеющие в данном случае смысла.

Как уже указывалось, величина $z_{0}$, вероятно, приблизительно постоянна для весьма обширных областей пространства. Не исключена возможность, что она приблизительно постоянна даже для всей Галактики. Тогда строение Галактики можно приближенно трактовать при допущении, что $z_{0}$ в точности постоянно. Возможно, что это соответствует действительности не хуже, чем допущения о стационарности и осевой симметрии Га- 
лактики, которые делаются в теории стационарной Галактики. Если считать величину $z_{0}$ постояннов̆, то условие (15) можно рассматривать, как дифференшиальное уравнение для потенциала. Решение этого уравнения известно из общей динамики $\left({ }^{25}\right)$. Оно имеет вид

$$
\Phi=\frac{\varphi\left(x_{1}\right)-\varphi\left(x_{2}\right)}{x_{1}^{2}-x_{2}^{2}},
$$

где $\varphi$ - произвольная функция, а $x_{1}^{2}$ и $x_{2}^{2}-$ корни уравнения

$$
\frac{R^{2}}{x^{2}-z_{0}^{2}}+\frac{z^{2}}{x^{2}}=1 \text {. }
$$

K тому же результату, но несколько иным способом пришел Әддингтон в его указанной выше работе $\left({ }^{15}\right)$.

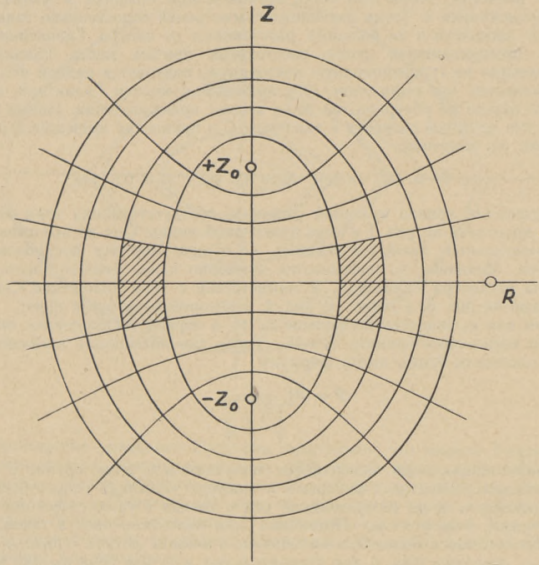

Puc, 3.

Как видно из уравнения (19), поверхности постоянных значений $x_{1}$ и $x_{2}$ являются софокусными поверхностями вращения второго порядка. Их общие фокусы расположены на галактической оси в точках с галактическим возвышением $+z_{0}$ и $-z_{0}$. Ряд меридианных сечений таких поверхностей изображен на рисунке 3 . Оказывается, что касательные плоскости к этим поверхностям наклонены к галактической плоскости на тот же угол, как наклонены в точке касания плоскости симметрии распределения скоростей, т. е. на угол $\alpha$. Поэтому оси эллипсондов скоро- 
стей должны быть перпендикулярными к этим поверхностям. Они являются, таким образом, «главными скоростными поверхностями», по терминологин Эллингтона $\left({ }^{15}\right)$. Вместе с тем эти поверхности, как можно показать, являются огибающими поверхностями звездных орбит. Область, в которой расположена орбита звезды, ограничена двумя эллипсоидами и двумя полостями двуполостного гиперболонда. (На рисунке 3 заштриховано сечение одной такой области.)

Возникает вопрос, мюжет ли потенциал Галактики соответствовать полученной выше формуле? Не приводит ли она к абсурдным результатам? Чтобы выяснить этот вопрос, мы приняли для $\varphi$ простое выражение

$$
\varphi=\Phi^{0} z_{0} \sqrt{x_{0}^{2}+x^{2}}
$$

где $\Phi^{0}$ и $x_{0}$ - постоянные. Выражение для функции $\varphi$ было выбрано с таким расчетом, чтобы она была четной и при больших $x$ возрастала пропорционально $x$. Тогда потенциал симметричен относительно галактической плоскости и на больших расстояниях от центра Галактики обратно пропорционален этому расстоянию, причем масса Галактики, умноженная на гравитационную постоянную, получается равной $\Phi^{0} z_{0}$.

Оказалось, что выражение (20) приводит к модели Галактики, которая не только не абсурдна, но даже весьма правдоподобна. Найдя формулы для круговой скорости и величины $C$ и привлекая имеющиеся о них данные, мы получили:

$$
\sqrt{\Phi^{0}}=425 \mathrm{kM} / \mathrm{ce \kappa}, z_{0}=3,6 \mathrm{k \Pi c}, x_{0}=0,6 \text { кпс. }
$$

Теоретическая кривая круговой скорости, соответствующая этим значениям, проведена на рис. 1 в виде пунктирной линии. Она не представляет наблюдательных данных в деталях, но общую картину воспроизводит хорошо. Величина $z_{0}$, оказывается примерно вдвое меньше расстояния Солнца от центра Галактики. В соответствии с этим положение Солнца нанесено на рис. 3 , а также на рис. 4 , о котором речь будет ниже.

Так как $x_{0}$ значительно меньше $z_{0}$, то в первом приближении оказывается возможным принять $x_{0}=0$. Тогда для потенциала в галактической плоскости будем иметь формулу

$$
\Phi=\Phi^{0} \frac{1}{\sqrt{1+\frac{R^{2}}{z_{0}^{2}}}} .
$$

Эквипотенциальные поверхности получаются в этом случае в виде сферических сегментов с центрами в фокусах «главных скоростных поверхностей», т. е, на галактической оси в точках с галактическими воз. вышениями $+z_{0}$ и $-z_{0}$. Потенциал для положительных $z$ таков, как если бы вся масса Галактики находилась в южном фокусе «главных скоростных поверхностей», а для отрицательных $z-$ как если бы она находилась в северном их фокусе. На рис. 4 изображено несколько меридианных сечений эквнпотенциальных поверхностей. Для $x_{0}=0$ они проведены прерывистыми линиями, а для вышепрннятого значения $x_{0}-$ сплошными линиями. Как видим, различие в значениях $x_{0}$ заметно повлияло на эквипотенциальные поверхности лишь вблизи галактической плоскости.

За исключением ближайших окрестностей галактической плоскости, потенциал завнсит в основном от поверхностной плотности Галактики, а поверхностная плотность, как мы видели, определяется в основном законом круговой скорости. Так как теоретическая кривая круговой скорости на рис. 1 не очень отличается от наблюденной, то можно думать, что 
общий внд сеченнй эквипотенциальных поверхностей на рис, 4. недалек от истнны. Интересно сравнить их с сечениями поверхностей равной плотности подснстемы короткопериоднческих цефенд по Б. В. Кукаркину $\left({ }^{3}\right)$. Некоторые из этих сечений проведены на рис. 4 пунктирными линиями. Если бы подсистема короткопериодических цефеид не вращалась и имела сферическое распределение скоростей, то поверхности равной плотности совпали бы у нее с эквипотенщиальными поверхностями. На самом же деле, как видно из рисунка, поверхности равной плотности значительно более сплюснуты. Причиной этого нужно в основном считать трехосность распределения скоростей. Вращение подсистемы может играть тут лишь второстепенную роль, поскольку оно слишком медленно.

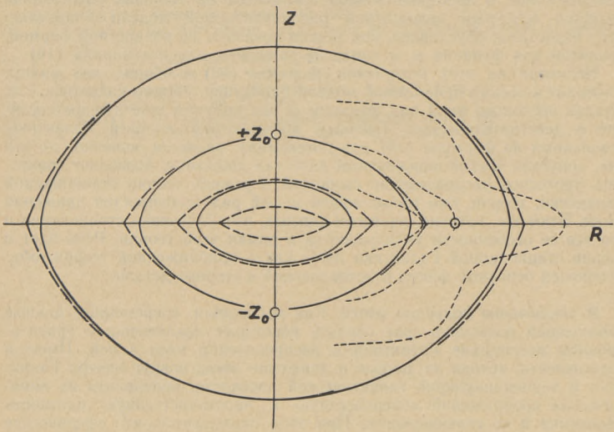

Рис. 4.

Применение уравнения Пуассона показывает, что модель Галактики, соответствуюшая формулам (18) и (20), имеет везде положительную плотность. Таким образом, она лишена основного недостатка модели Галактики П. П. Паренаго $\left(^{6}\right)$, которая приводит к отрицательным плотностям на больших расстояниях от галактической плоскости. Для малых $x_{0}$ и не очень больших по модулю $z$ формулы (18) н (20) дают следующее выраженне для плотности:

$$
4 \pi G_{\varrho}=\frac{\Phi_{0}}{z_{0} x_{0}} \frac{1}{\sqrt{1+\frac{R^{2}}{z_{0}^{2}}\left(1+\frac{R^{2}}{z_{0}^{2}}+\frac{z^{2}}{x_{0}^{2}}\right)^{1 / 2}}} .
$$

Поверхности равной плотности согласно этой формуле приблизительно сфероидальны. Отношение полуосей в окрестностях центра Галактики равно $\frac{2}{\sqrt{3}} \frac{x_{0}}{z_{0}}$. При увеличении расстояния от центра оно очень медленно возрастает. Если взять для $x_{0}$ и $z_{0}$ вышеприведенные значения, то отно- 
шение полуосей получается в среднем равным примерно 1/5. Для поверхностной плотности при малых $x_{0}$ получается выражение

$$
2 \pi G \delta=\frac{\Phi^{0}}{z_{0}} \frac{1}{\left(1+\frac{R^{2}}{z_{0}^{2}}\right)^{\pi / 2}} .
$$

Кривая поверхностной плотности, соответствующая принятым значениям для $\Phi^{0}$ и $z_{0}$, проведена в виде пунктирной линии на рис. 2. Она, в общем, хорошо воспроизводит кривую, полученную из наблюдательных данных. Лишь для больших $R$ поверхностная плотность убывает неправдоподобно медленно (пропорционально $R^{-3}$ ). Слишком медленное падение поверхностной и пространственной плотностей на больших расстояниях является основным недостатком рассматриваемой модели Галактики. Этот недостаток обусловлен, как можно показать, не конкретной формой, принятой для функции $\varphi$, а самим выражением для потенциала (18).

Несмотря на этот недостаток, формула (18) в общем, как видим, приводит к весьма прнемлемой модели Галактики. Можно надеяться, что, придав несколько иной вид функции $\varphi$, мы получим еще лучшее согласне с действительностью. Главным достоинством моделей Галактики, основанных на формуле (18) для потенциала, является, конечно, то, что она, допуская третий однозначный интеграл движения, позволяет трактовать трехосное распределение скоростей в рамках теории стационарной Галактики, теории уже объяснившей целый ряд особенностей движения звезд. Этим мы, конечно, не хотим сказать, что Галактика стационарна и что все ее особенности укладываются в рамки этой теории. Речь идет о теории стационарной Галактики лишь как о приближенной теории, объясняющей основные факты и оставляющей в стороне детали.

В заключение подведем итоги. Как мы видели, современные данные наблюдений позволяют уже сделать некоторые заключения о гравитационном потенциале Галактики и распределении масс в ней. Имеется возможность, исходя из данных о движении звезд вокруг центра Галактики и перпендикулярно галактической плоскости, определить из динамических соображений поверхностную и пространственную плотности Галактики и ее сплющенность. При этом оказывается, что сферические подсистемы Галактики играют заметную роль в ее динамике. Далее оказывается, что линдбладовский закон галактического вращения не подтверждается ни наблюдениями, ни теорией и что скорость освобождения значительно больше, чем в последнее время принималось. Факт трехосности распределения скоростей звезд позволяет сделать дополнительные заключения о возможном строении Галактики. Вводя третин̈ интеграл движения звезд, мы можем объяснить трехосное распределение скоростей в рамках теории стационарной Галактики, причем получаем модель Галактики, в общих чертах согласующуюся с данными наблюдений.

Институт физики и астрономии Академии наук Эстонской ССР

Поступила в редакщию 23 VII 1953

\section{ЛИТЕРАТУРА}

1. Г. Г. К уз мин, Публ. Тартуск, астр. обс., 32, 5, 1952,

2. Г. Г. К у з н н, Публ. Тартуск, астр. обс., $32,211,1952$.

3. Б. В. К ука ркин, Исследование строения и развития звездных систем на основс изучения переменных звезд, Гостехнздат, М.-Л., 1949.

4. П. П. П а рен а г о, Успехи астр. наук, 4, 69, 1948. 
5. П. П. Паренаго, Астр. журн., 27, 329, 1950.

6. П. П. Парена го, Астр. журн., 29, 245, 1952.

7. П. П. Парен аго, Динамнка звездных систем, Курс звездной астрономин, 2-е изд. Гостехиздат, М.-Л., 1946.

8. П. П. П а рен а го, Перем, звезды, 6, 102, 1948.

9. П. П. П а рен а го, Астр. журн., $27,150,1950$.

10. П. П. Парен а го, Сообш. Гос. астр. ннст. Штернберra, 30, 3, 1949.

11. С. Б. Пикельнер, Докл. Акад. наук СССР, 88, 229, 1953.

12. В. С. С а фронов, Астр, журн., 29, 198, 1952.

13. K. F. B ottling er, Erg. d. exakt. Naturwiss. 11, 31, 1932.

14. S. Chandrasekhar, Monthly Not. R. A. S., 98, $710,1938$.

15. A. S. Edding to n, Monthly Not. R. A. S., 76, 37, 1915.

16. J. H. Jea ns, Monthly Not. R. A. S., $82,122,1922$.

17. J. H. Jean s, Monthly Not. R. A. S., 76, 70, 1915.

18. B. Lindblad, Arkiv f. Mat. Astr. o. Fys., 20A, No 17, 1927.

19. B. L in d b l a d, Bergstrand Festskr., 15, 1938.

20. B. L i nd bla d, Die Milchstrasse, Die Dynamik der Milchstrasse, Handb. d. Astroph., $5 / 2,1933$

21. J. H. O ort, Bull. Astr. Inst. Neth., 4, 269, 1928.

22. J. H. O ort, Bull. Astr. Inst. Neth., 6, 249, 1932.

23. L. Pere k, Contr. Astr. Inst. Masaryk Univ., 1, No 6, 1948.

24. J. S chilt. Astr. Journ., 55, 97, 1950.

25. E. T. Whittaker, Analytical Dynamics, Cambridge, 1904, $\$ 152$.

26. A. B. W y se, N. U. M a y a I I, Astroph. Journ., 95, 24, 1942. 